\title{
Les emplois de proximité: Une opportunité de professionnalisation pour les femmes de milieu populaire?
}

\author{
ANNIE DUSSUET \\ LESTAMP \\ Département de Sociologie \\ Université de Nantes
}

Face à la nécessité grandissante, éprouvée au cours des années 80, de lutter contre le chômage, les pouvoirs publics se sont trouvés amenés à faire l'inventaire des "gisements d'emploin possibles. Dans cette quête, l'intérêt s'est rapidement porté sur le secteur des services qui paraissait à la fois répondre à des besoins quasi infinis, réaliser de faibles gains de productivité ${ }^{1}$ et être difficilement délocalisable. Mais dans ce tertiaire si hétérogène, les services "de proximitén semblaient présenter à un degré supérieur ces caractéristiques.

Que sont en effet les "services de proximitén? Bien que leurs contours soient mal définis ${ }^{2}$, ils désignent tous des services habituellement rendus gratuitement dans le cadre de solidarités familiales ou de voisinage. Leur caractère hétéroclite et leur ancrage local, difficiles à assumer par l'industrialisation, deviennent alors promesses d'emplois. De plus ces services, parce qu'ils sont habituellement réalisés bénévolement, sur un mode familial ou amical, semblent ne nécessiter aucune formation préalable. Ils apparaissent donc comme une source rêvée d'emplois pour des chômeurs ou plutôt des chômeuses évincées du marché du travail par leur manque de qualification. La Commission Européenne en distingue 4 grands types: les services de la vie quotidienne, les services d'amélioration du cadre de vie, les services culturels et de loisirs et les services de protection de l'environnement. Nous nous intéresserons ici à la $1^{\text {ère }}$ catégorie: les services d'aide à la vie quotidienne, parmi lesquels nous distinguerons encore les services «à domicile" d'autres, produits hors du domicile.

1 Voir toutefois la critique de cette notion effectuée par Gadrey (1996).

2 Les emplois de proximité désignent un ensemble d'emplois de service adans et autour de l'habitation", destinés à pallier au relâchement des solidarités de voisinage, ou bien encore accompagnant la vente de biens aux consommateurs. On y trouve donc pêle-mêle les livraisons d'achats à domicile, les petites réparations, les travaux d'entretien du logement, l'accompagnement d'enfants ou de personnes âgées... 
De nombreux rapports ${ }^{3}$ soulignaient ainsi qu'un nombre non négligeable de création d'emplois pouvait être espéré de leur développement. C'est à partir de ce raisonnement que les responsables politiques ont, depuis le milieu des années 80 , cherché à favoriser ces emplois grâce à des mesures d'exonération fiscale pour les ménages utilisateurs et des simplifications administratives. C'est d'abord une loi de janvier $1987^{4}$ qui exonère de charges sociales les employeurs particuliers de plus de 70 ans, puis une mesure de décembre 1991 instaurant une réduction d'impôt sur le revenu de $50 \%$ des sommes versées pour l'emploi d'un salarié travaillant à la résidence du contribuable (emplois dits "familiaux"). C'est ensuite la loi de décembre 93 instaurant le chèque-emploi-service, qui permet de simplifier pour les employeurs la déclaration et le paiement des cotisations sociales (un simple chèque suffit pour faire tout cela). Le résultat de cette politique est important puisqu'on considère que 93000 salariés supplémentaires ont intégré ce type d'emploi entre 91 et 96 , même s'il est difficile de distinguer un surplus net d'emplois d'éventuels effets d'aubaine, et même si la plupart de ces emplois sont à temps partiel.

Il faut noter surtout que ces services de proximité d'aide à la vie quotidienne sont largement féminisés, à $99 \%$ selon une étude du Ministère du travail de 1995 (Zilberman 1995). Tous ces emplois réalisent donc une sorte de chassé-croisé entre des femmes qui, pour pouvoir mieux répondre aux exigences de leur propre carrière professionnelle, payent les services d'autres femmes qui les remplaceront dans leurs tâches domestiques. On peut s'interroger sur les conséquences pour les femmes ainsi employées de ces évolutions. En effet, "faire des ménages", c'est à dire se déplacer au domicile d'autrui pour y effectuer les gestes d'entretien courant de la maison et du linge constitue depuis longtemps pour les femmes des milieux populaires démunies de qualification une manière d'entrer sur le marché du travail salarié. Pour bon nombre d'entre elles, la similitude avec les gestes du travail domestique effectué dans leur propre foyer peut apparaître comme une solution à leur anemployabilitén. Il s'agirait en quelque sorte de réaliser contre salaire le même travail qu'elles effectuent chez elles, gratuitement. Mais le travail domestique, travail gratuit accompli par les femmes chez elles pour les membres de leur famille obéit à des "logiques" propres 5, et qui le définissent en opposition avec

3 Voir par exemple: Greffe (1990), ou, plus récemment Bailly (1996) et Conseil d'Analyse Economique (1998).

4 Pour plus de précision sur ces mesures, voir Causse, Fournier et Labruyère (1998).

5 Des "logiques" que nous avons mises en évidences dans des recherches antérieures (Dussuet 1997). 
le travail salarié. Comment, dès lors, le travail domestique subit-il cette externalisation par la salarisation? A quel type d'emplois donne-t-il naissance? Ceux-ci permettent-ils aux femmes de sortir du confinement domestique? Aboutissent-ils à revaloriser des tâches longtemps considérées comme sans intérêt? Peuvent-ils constituer une "profession"?

Cela paraît peu probable car les emplois de service de proximité d'aide à la vie quotidienne sont parmi les plus dévalorisés qui soient. Faut-il alors interpréter le nouveau dynamisme de croissance de ces emplois comme un retour à des formes d'emplois "serviles"? Le caractère "domestique" du travail effectué conduit-il inéluctablement les femmes à retrouver des formes de sujétion caractéristiques de la condition ancillaire?

Ces questions prennent toute leur importance dans la période actuelle, où, en France, l'extension du chômage amène de nombreux acteurs, publics et privés à se saisir de cette question. De nombreuses associations ${ }^{6}$ en particulier proposent ce type de services. La plupart sont regroupées en fédérations nationales et toutes n'ont pas les mêmes objectifs ni la même histoire: certaines s'affirment comme associations familiales", d'autres sont des émanations des collectivités locales, certaines visent l'insertion d'une population marginalisée sur le marché du travail, d'autres mettent en avant la solidarité vis à vis des personnes dépendantes. L'éventail est donc très large mais nous nous sommes intéressées ici essentiellement aux associations proposant un service ménager (entretien du logement, du linge, préparation de repas), à l'exclusion de celles qui se sont spécialisées dans la garde d'enfants ${ }^{7}$.

C'est donc à travers l'analyse des premiers résultats d'une enquête menée depuis 2 ans dans les agglomérations d'Angers et de Nantes auprès de responsables et de salariées d'associations offrant des services de proximité dans le domaine ménager que nous tenterons d'évaluer les enjeux, pour les femmes employées, de la croissance de ces emplois d'aide à la vie quotidienne.

\section{DES EMPLOIS POUR LES CHÔMEUSES}

Parmi les emplois de proximité, ce sont en effet ces services d'aide à la vie quotidienne qui sont apparus comme les plus prometteurs en ter-

6 Par exemple, pour l'agglomération d'Angers (200 000 habitants), 15 structures différentes recensées par un guide paru en 1997.

7 Nous ne nous intéresserons aux services de prise en charge des enfants que quand ils s'ajoutent au travail ménager, sans en être la composante principale, les services de garde d'enfants posant des problèmes assez différents en termes d'organisation. 
mes d'emplois. D'une part, ils semblaient répondre à une importante demande de la part de ménage composés de "double-actifs", trop absorbés par leurs tâches professionnelles pour faire face au travail domestique quotidien, d'autre part, ils apparaissaient comme faciles d'accès pour certains demandeurs d'emploi particulièrement difficiles à insérer, à savoir les chômeuses faiblement, ou non-qualifiées.

\subsection{La redécouverte du travail domestique}

Ainsi, d'une manière qui n'avait certes pas été prévue par les féministes des années 70 , les responsables politiques ont récemment redécouvert, en France, le travail domestique, en tant que créateur de richesse.

\subsubsection{Une véritable "production" domestique}

En effet, dans les sociétés occidentales modernes, les femmes réalisent encore gratuitement au sein de la famille, du fait de la division des tâches entre les sexes, une "création d'utilitén qui permet l'entretien de la maisonnée. Même si ces tâches ont pu être allégées par l'amélioration de l'équipement des logements, elles n'ont pas été absorbées par l'industrialisation: ainsi que le montre par exemple, pour la France, la dernière enquête budget-temps réalisée par l'INSEE. Les ménages y consacrent encore un temps considérable. De plus ce sont toujours essentiellement les femmes (Brousse 1999) qui le prennent en charge (5h de travail quotidien en moyenne, contre $2 \mathrm{~h} 30$ pour les hommes).

Cette production de richesses est largement occultée dans les statistiques économiques. Pour les comptables nationaux, l'opération de "production" est uniquement référée au marché. Ne sont donc inclus dans l'agrégat Produit Intérieur Brut (PIB) que les biens et services produits à l'aide de facteurs de production acquis sur le marché, ou bien destinés à y être délivrés. Tout ce qui entre dans le ménage à fin de consommation est donc considéré comme consommé sans qu'aucune valeur n'y soit ajoutée: le travail domestique qui consiste à préparer les biens pour la consommation (cuisine, par exemple) ou à les entretenir, pour permettre une durée d'utilisation plus longue (ménage, lessive, etc...) est improductif de ce point de vue, puisque d'une part il n'est pas rémunéré (à la différence, donc, des mêmes tâches réalisées par une employée de maison, qui elles, sont considérées comme productives) et que d'autre part il n'est pas destiné à être cédé sur le marché (comme le seraient, par exemple, 
les conserves ou confitures réalisées par une femme d'agriculteur et destinées à être vendues à la ferme).

$\mathrm{Au}$ cours des années 70 , les mouvements féministes avaient déjà attiré l'attention sur ce travail, réalisé essentiellement par les femmes, avec des objectifs divergents: pour les Italiennes par exemple, il s'agissait plutôt de réclamer que ce travail soit rémunéré, afin de garantir aux femmes "au foyer" un revenu propre qui assure leur indépendance (voir en particulier les textes collectifs publiés par M. Dalla Costa et S. James 1973); pour les Françaises, l'objectif était plutôt de faire reconnaitre ce travail et d'en permettre la socialisation à travers la création de places de crèches ou de services collectifs de laverie par exemple.

Dans ce contexte, des chercheuses vont démontrer statistiquement la réalité de la richesse produite par le travail domestique. Ainsi, dans le cadre de la comptabilité nationale, Ann Chadeau et Annie Fouquet (1981a et 1981b) distinguent parmi les activités domestiques celles qui sont "productives": ce sont celles "qui créent des biens ou des services qui auraient pu être fournis par une autre unité économique. Faire la cuisine ou le ménage sont des activités productives car quelqu'un pourrait être engagé pour effectuer ces tâches; regarder la télévision et dormir ne sont pas des activités productives car elles ne peuvent être réalisées par une tierce partie.

Nous pouvons les suivre pour définir ce que nous entendons précisément par "travail domestique». Il s'agit du "travail effectué gratuitement pour l'entretien du groupe familial. Cette définition large recouvre des tâches concrètes aussi différentes que "préparer les repas", "faire la vaisselle", "nettoyer les vitres", "passer l'aspirateur", mais aussi "faire les courses", ou encore "baigner les enfants", "surveiller leur travail scolaire", "les emmener chez le dentiste.. On voit par cette énumération que le travail domestique ne désigne pas seulement des "âches ménagères" mais aussi relationnelles et éducatives. Le terme "domestique" n'est pas non plus limité ici à un sens spatial: le travail "domestique" se déroule aussi à l'extérieur de la maison. Sa caractéristique essentielle réside dans sa gratuité et donc dans le rapport social particulier dans lequel il est effectué. Il suppose entre le travailleur (ou plutôt la travailleuse) et les bénéficiaires du travail un lien préexistant, de type affectif, qui permet d'expliquer la gratuité de l'échange.

Mais il faut aussi relever l'importance considérable de ce travail révélée par les résultats de la recherche statistique: pour la France, la valeur du "produit domestique" oscille entre 32 et $77 \%$ du PIB marchand, selon les modes d'évaluation retenus ${ }^{8}$.

8 Pour des précisions concernant les différents modes d'évaluation, voir Chadeau et Fouquet, op. cit. ou Chadeau (1992). 


\subsection{2. ...à "externaliser"}

On peut se demander comment une production d'une telle ampleur a pu si longtemps échapper au marché. Les contraintes liées à la diversité des exigences des consommateurs, au caractère personnalisé du service attendu en fournissent sans doute une bonne explication. Il faut aussi remarquer que la frontière entre services fournis par le marché et services produits de façon domestique varie considérablement dans le temps. D'ores et déjà, les fonctions éducatives par exemple ou encore la préparation de certains repas sont largement prises en charge sinon de façon marchande, du moins de façon externe aux cellules domestiques.

Mais l'extension du chômage amène les responsables politiques à la recherche de agisements d'emploin à tenter d'élargir ce processus. Le travail domestique, travail ahors emploin, qui avait jusqu'alors permis, par la mobilisation gratuite des femmes, la satisfaction de multiples besoins matériels et relationnels, qu'il s'agisse de garde d'enfants, de soutien scolaire, de soins quotidiens aux malades et aux personnes âgées, ou bien encore, tout simplement, de ménage, de cuisine et de repassage, apparaît alors comme un concurrent du travail salarié. Toutes ces activités vont donc être envisagées comme des sources d'emploi potentiel: il va s'agir de faire "Sortir" les tâches domestiques de l'espace et (ou) du rapport social domestiques, et, sous quelque forme que ce soit, de réaliser ce que nous nommerons leur "externalisation. Ainsi voit-on se multiplier depuis la fin des années 80 des emplois qui, intitulés "emplois de proximitén, "emplois familiaux", "services à domicile" opèrent tous, sous des formes diverses, une "externalisation" de travail domestique, dans un sens non exclusivement spatial, puisque les services échangés sont souvent réalisés à domicile. Mais ils sont pris en charge de façon externe, et surtout, le plus souvent payante.

Plusieurs formes d'externalisation peuvent être inventoriées. Tantôt celleci se fait par l'embauche d'un(e) salarié(e) qui intervient à domicile: c'est la forme dite du ugré à grém. On peut noter au passage que cette forme s'affranchit parfois des impératifs de la formalisation de l'emploi. On trouve dans ce cadre beaucoup de travail dit "au noir" et, même quand les salariées sont "déclarées", les règles du droit de travail ne sont pas pour autant toujours appliquées. Il existe aussi dans ce secteur de nombreuses associations qui jouent le rôle d'intermédiaires entre les salariées et les utilisateurs soit parce qu'elles sont "prestataires" (elles sont alors employeuses et vendent un "service" aux consommateurs), soit parce qu'elles ont un statut de "mandataire" (elles s'occupent alors en lieu et place de l'utilisa- 
teur, qui reste le véritable employeur, de toutes les formalités liées à l'emploi), soit encore parce que, de façon dérogatoire au droit du travail, elles ont obtenu de faire du prêt de main d'œuvre (c'est le cas des "associations intermédiaires", créées en 1987, et destinées à permettre l'insertion sur le marché du travail de chômeurs particulièrement éloignés des critères d'emploi), soit enfin ces associations créent des structures de production et de vente de services dans un lieu différent des domiciles privés des consommateurs (c'est le cas surtout pour les tâches d'entretien du linge: nettoyage, repassage, couture), le lien entre les salariées et les utilisateurs de leur service étant ici le plus distendu.

\subsection{Des caractéristiques féminines d'emploi}

Mais quelle qu'en soit la forme, les emplois de proximité de services d'aide à la vie quotidienne sont, on l'a dit, particulièrement féminisés; ils sont occupés à $99 \%$ par des femmes. Au delà de ce constat numérique, on pourrait dire que ce sont des eemplois féminins" tant ils offrent un concentré des caractéristiques communes à la majorité des emplois occupés aujourd'hui par les femmes ${ }^{9}$.

\subsubsection{Une image féminine}

Ce sont d'abord des emplois féminins au sens où seules des femmes semblent pouvoir s'y intégrer. Outre la quasi absence des hommes dans ces emplois, on peut noter la difficulté des quelques individus masculins qui s'essaient à cette véritable transgression à se faire accepter, les femmes semblant les seules à pouvoir accomplir cette intrusion dans l'univers domestique et intime des utilisateurs. Il s'agit bien d'un atravail de femme" au sens plein du terme, c'est à dire accompli quasi exclusivement par des femmes d'une part, et dont les représentations sont construites autour d'une figure féminine.

Quel est donc le contenu de ces emplois? Plusieurs appellations peuvent être relevées, qui correspondent à des "métiers" différents ${ }^{10}$. Parmi

9 Nous retrouvons ici les résultats présentés au niveau européen par Maria Jepsen et Danièle Meulders (1998) qui montrent la mauvaise qualité des emplois générés pour les femmes par les sinitiatives locales d'emploi, en termes de statut professionnel, de stabilité, et de pourcentage de temps partiel.

10 Les «travailleuses familiales" récemment rebaptisées «techniciennes d'intervention sociale et familiale* doivent être considérées à part, car même si elles interviennent 
les salariées qui interviennent à domicile on trouve bien sûr des afemmes de ménagen, qui accomplissent surtout des tâches d'entretien, mais aussi des "aides à domicile", intervenant plutôt auprès de personnes âgées, des "auxiliaires de vie" ou "garde-malade", plus spécialisées dans l'aide à des personnes malades ou handicapées. Toutefois, ces catégories ne sont pas étanches et si ces femmes accomplissent des tâches fort diverses, du ménage à la toilette de personnes âgées, en passant par le repassage et la garde d'enfants, elles ont toutes comme caractéristique commune de se substituer à celles accomplies habituellement gratuitement par les femmes en tant que filles, épouses et(ou) mères sur le mode du devoir imposé par les relations de parenté.

Il s'agit donc bien de remplacer des femmes par d'autres femmes, retrouvant ainsi, dans un cadre salarié, le cercle de l'échange de services entre femmes dans la famille étendue, bien montré par D. Chabaud, D. Fougeyrollas, et F. Sonthnonnax (1985).

\subsubsection{Des conditions d'emploi précaires}

Mais comme beaucoup d'emplois féminins aujourd'hui ${ }^{11}$, ces emplois sont aussi des emplois précaires, cette précarité semblant plutôt se renforcer dans les années récentes. Le plus souvent en effet, les salariées des emplois de proximité de la vie quotidienne ont affaire à des employeurs multiples. Chaque salariée a, en moyenne, entre 1,6 et 1,7 employeur déclaré, chiffre en augmentation régulière depuis 1992 (Céalis et Zilberman 1997); cela provient de la forme de contrat qui a été encouragée par les pouvoirs publics ces dernières années, le "gré à grén, où l'utilisateur est l'employeur direct de la salariée. On retrouve là une forme ancienne de relation d'emploi puisque c'était celle de la domesticité, par laquelle beaucoup de jeunes filles entraient sur le marché du travail, du siècle dernier aux années 60 . Mais le sens de cette forme d'emploi a changé. Si les "domestiques" du début du siècle, les "bonnes à tout faire" et encore les "employées de maison" des années plus récentes avaient en général un employeur unique qui les utilisait à plein temps (et même au

aussi à domicile pour réaliser des tâches ménagères, à la place de la mère de famille défaillante le plus souvent, leur action comporte un volet éducatif de aremise en ordre" de la famille qui n'existe pas dans les autres cas et qui est reconnu à travers leur formation professionnelle sanctionnée par un diplôme.

11 Sur la précarité plus grande de l'emploi féminin en général, voir par exemple Maruani (1998). 
delà!), les logeait et les nourrissait, les femmes de ménage ou aides à domicile d'aujourd'hui ne travaillent que quelques heures par semaine pour un employeur, ce qui les oblige à multiplier les utilisateurs-employeurs. Le lien de sujétion, très fort autrefois entre patron et salariée en est certes allégé, mais dans le même temps, la sécurité de ce lien s'est évaporée, la salariée étant beaucoup plus soumise aujourd'hui aux risques biographiques de son employeur (modification de la taille de la famille ou du logement, rythme d'activité professionnelle de la "maîtresse de maison", et surtout risques d'hospitalisation, ou même de décès dans le cas d'employeurs âgés).

Les mesures gouvernementales récentes ont pourtant favorisé ce type d'emploi. La loi du 27 janvier 87, en instaurant une exonération de charges sociales pour les particuliers-employeurs de plus de 70 ans, a rendu plus attractive pour cette catégorie d'utilisateurs potentiels la formule de l'emploi direct en "gré à gré" par rapport au recours à une association prestataire d'aide à domicile. La conséquence de cette mesure a été le développement au sein de ces mêmes associations - jusque là employeuses- d'organismes "mandataires" qui mettent en rapport employeurs particuliers et salariées et assurent certaines formalités administratives, mais n'emploient plus eux-mêmes les salariées. Cela a contribué à renforcer la précarité des emplois puisque les postes créés par des particuliers (d'autant plus quand il s'agit de personnes âgées) apparaissent beaucoup moins stables que ceux qui peuvent l'être par des organismes collectifs.

Les liens d'obligation de l'employeur vis à vis du salarié instaurés par le contrat de travail se sont aussi distendus avec l'apparition, en 1993 du "chèque-emploi-service" qui représente une modalité d'emploi encore moins contraignante pour l'employeur puisque son utilisation ne préjuge en rien de procédures d'embauche ou de licenciement ${ }^{12}$.

Les emplois qui se sont développés dans ce secteur ont donc une stabilité très faible. Certaines salariées employées par l'intermédiaire d'associations mandataires peuvent ainsi, en l'espace de quelques années avoir accumulé plusieurs centaines de contrats de travail.

12 La nature du contrat de travail institué par l'utilisation du chèque emploi-service est en effet peu claire. S'agit-il de contrat à durée déterminée (CDD) ou à durée indéterminée (CDI)? Les utilisateurs en tout cas ne semblent guère conscients des engagements qu'ils prennent par l'intermédiaire de ce chèque à l'égard de leurs salariées. Voir sur ce point la discussion de Kerbourch (1997). 


\subsubsection{Des emplois à temps partiel}

Cette multiplication des employeurs n'est possible que parce que la durée des prestations pour chacun d'eux est faible. Même en totalisant les interventions chez les divers employeurs dans une même période, les emplois de proximité d'aide à la vie quotidienne restent des emplois à temps partiel, à temps très partiel même, puisque la durée hebdomadaire de travail y était de $8 \mathrm{~h}$ en moyenne en 1996. Mais pour les $2 / 3$ des salariées, cette durée était inférieure à 6 heures, une proportion très faible (12\%) ayant une durée totale d'emploi supérieure au mi-temps (Céalis et Zilberman 1997; Zilberman 1995).

Il faut donc nettement distinguer entre le nombre d'emplois créés dans ce secteur, +500000 entre 91 et 96 , chiffre le plus souvent avancé par les politiques, le nombre de salariées supplémentaires, beaucoup plus modeste, et le nombre d'équivalents temps pleins, environ 80000 . Ce phénomène d'émiettement est encore plus sensible dans le cas où les employeurs recourent au chèque emploi-service et surtout quand les Associations Intermédiaires sont employeuses. Dans ces dernières structures, environ $29 \%$ des salariés à domicile ont travaillé moins de 30 heures dans le trimestre de référence (Céalis 1997), soit à peine 3 heures par semaine. On peut objecter à ce dernier constat que le rôle des Associations Intermédiaires est conçu comme temporaire: leur objectif est de permettre aux salariées de reprendre pied progressivement sur le marché de l'emploi. Mais l'étude de la DARES montre que pour près de la moitié des salariés, et surtout pour les femmes, l'Association Intermédiaire constitue un lieu durable d'activité. Même si la durée de travail hebdomadaire de ces "ancienn(ne)s" dans la structure est en général un peu plus longue (le nombre de missions confiées est plus important), elle reste le plus souvent largement inférieure à un mi-temps.

Ces constats nationaux recoupent largement les observations que nous avons pu faire dans les structures associatives étudiées. Dans les associations d'insertion, parvenir à fournir à certaines salariées un horaire à mitemps semble proche de l'exploit. Ce n'est que dans des associations plus anciennes, dites "d'aide à domicilen, et structurées autour de l'emploi de "travailleuses familiales" (aujourd'hui considérées comme "travailleuses sociales" au même titre que les éducateurs ou les assistantes sociales par exemple, les tâches ménagères n'étant qu'un support à leur action éducative) (Bonamy 1997) ou bien encore dans des structures prestataires publiques (Centres Communaux d'Action Sociale - CCAS) que les aides à domicile atteignent des horaires à temps plein. Il semble donc que l'on 
ait ici affaire à un type d'emploi structuré comme emploi à temps partiel: ces emplois sont perçus par tous les acteurs, responsables associatifs et même salariées, comme des emplois à temps partiel "par nature".

Il est intéressant d'essayer de comprendre cette impossibilité du temps plein, en partant des arguments invoqués par les acteurs eux-mêmes. Le premier problème est celui du type d'horaires demandé aux salariées intervenant dans les domiciles privés. Ainsi par exemple, réclame-t-on souvent aux femmes de ménage d'effectuer leur tâche en dehors de la présence des membres de la famille utilisatrice, pendant les horaires de travail professionnel de ceux-ci. C'est une contrainte forte pour l'organisation du travail des salariées qui interviennent souvent, on l'a vu, chez plusieurs clients ou employeurs. Ainsi, alors que dans les bureaux et les collectivités, les horaires de travail des femmes de ménage sont le plus souvent décalés avant l'ouverture ou après la fermeture, les plages horaires utilisables par les femmes de ménage en emplois familiaux se réduisent au milieu de journée. Comme l'explique une salariée:

les après-midi, je fais 3 heures, beaucoup de gens demandent $3 \mathrm{~h}$, mais avant, je me rappelle, à temps plein, je travaillais jusqu'à $19 \mathrm{~h}$. Alors, 3 heures, l'après-midi, de 14 à $17 \mathrm{~h}$, ça fait encore 2 heures, de 17 à $19 \mathrm{~h}$ !

Mais dans ce type d'emploi, elle ne trouve personne qui souhaite son intervention entre 17 et 19 heures.

De plus, elles doivent aussi s'ajuster au rythme de la vie familiale des utilisateurs: semaine, week-ends, jours de congés des enfants. Il y a ainsi une demande très forte pour des interventions le lundi et le vendredi, très peu par contre pour le mercredi. Si l'on ajoute la nécessité de se déplacer entre les différents domiciles, il devient quasi impossible de composer un planning de travail à temps plein. La situation est semblable, même si les contraintes s'expriment différemment, pour les aides à domicile qui interviennent chez des personnes âgées ou handicapées. La présence devient dans leur cas une composante essentielle du service attendu et peut rendre nécessaire une intervention plus fractionnée, ou incluant des horaires tardifs ou de week-end (présence souhaitée pour le coucher des personnes par exemple). Il s'agit encore de contraintes supplémentaires pour les salariées, les empêchant d'accéder au plein temps en même temps qu'elles interfèrent avec leurs propres rythmes sociaux.

Lors des entretiens réalisés, plusieurs salariées ont expliqué longuement cette inaccessibilité du temps plein, en la regrettant le plus souvent:

Moi, si on me trouve $6 \mathrm{~h}$ à faire d'affilée, je le fais, hein, même un temps complet, s'ils me trouvent un temps complet le mercredi, moi, je le fais. 
Il faut pourtant relever que les obligations familiales et domestiques des salariées elles-mêmes contribuent à cette obligation d'exercice à temps partiel. Parmi les femmes rencontrées, celles qui sont mères de jeunes enfants cumulent avec les exigences de leurs clients leurs propres contraintes domestiques. Par exemple, Madame A. travaille chez un client le matin, un autre l'après-midi, tous les jours de la semaine sauf le mercredi:

$2 \mathrm{~h}$ ou $3 \mathrm{~h}$, ça dépend, jamais moins de $2 \mathrm{~h}$ et... moi, je ne prends pas plus de $3 \mathrm{~h}$. Moi, j'ai les horaires d'école pour les enfants, ça me fait de $9 \mathrm{~h}$ le matin, jusqu'à 16h30 le soir, $9 \mathrm{~h}-12 \mathrm{~h}, 13 \mathrm{~h} 30-16 \mathrm{~h} 30$, ça correspond aux horaires d'école.

Malgré son souhait de travailler plus (et d'avoir un salaire plus élevé), elle ne peut cumuler plus d'interventions car cela l'obligerait à rechercher une solution de garde périscolaire pour ses enfants. Au total, elle ne peut donc guère assurer que 18 heures par semaine, soit à peine un mi-temps.

Sensibles à cet ensemble de contraintes, les responsables associatifs jugent donc que, sauf exception, il est irréaliste de proposer des emplois à plein temps dans ce secteur et les emplois familiaux sont ainsi constitués structurellement comme emplois à temps partiel. Cela participe au marquage féminin de cette situation d'emploi car seules des femmes peuvent se contenter de quelques heures de travail par semaine, du fait de leur légitimité moins établie dans l'emploi. Elles les acceptent donc, dans une perspective traditionnelle de "complément" de salaire, même si beaucoup de femmes occupant ces emplois sont en réalité seules ou en situation monoparentale.

\subsubsection{Une qualification faible}

Enfin, ces emplois se signalent par leur qualification nulle, ou presque. C'est même, on l'a dit, cette caractéristique qui a attiré l'attention des responsables politiques. Il semble que n'importe quelle femme puisse accomplir les tâches demandées, pour la bonne raison qu'elle les accomplit déjà, en règle générale, dans son propre cercle familial, en tant qu'épouse, mère ou fille. Des "qualités féminines" semblent suffisantes pour occuper ces emplois, sans qu'il soit nécessaire d'être "qualifiéen, la différence entre les deux notions étant bien sûr dans la reconnaissance sociale attachée à la seconde, à la différence de la première.

On peut voir ici à l'œuvre le même processus de dénégation de la qualification que celui déjà signalé dans les années 60 par Madeleine Guilbert à propos des postes de travail féminins dans l'industrie: celle-ci notait en effet comment les employeurs préféraient des femmes sur cer- 
tains postes nécessitant rapidité et minutie, et comment ces aptitudes spécifiques prêtées aux femmes devaient être rapprochées de leurs tâches domestiques:

Dans les deux cas, la même prédominance des mouvements mettant en jeu les bras, les avant-bras, ou les mains, leur amplitude généralement faible. Dans les deux cas, le chevauchement ou la coordination des gestes des deux mains sont des facteurs importants de l'accélération du travail. La capacité de rapidité des femmes dans les travaux industriels qui leur sont confiés s'explique dès lors en partie par les aptitudes gestuelles acquises dans la pratique des travaux ménagers (Guilbert 1966: 213-214).

Les qualités alors décrites comme "féminines" et pensées comme naturelles ne sont donc bien sûr que le résultat de la socialisation féminine aux tâches domestiques. Mais cette "naturalisation" des qualités féminines permet de les envisager comme "aptitudes" et non comme "savoir-faire", ainsi que l'a aussi montré Danièle Kergoat à propos des infirmières. Ce rejet vers la nature autorise l'absence de rémunération de ce qui n'est perçu, par les femmes elles-mêmes, que comme une manifestation de leur féminité (Kergoat, Imbert, Le Doaré et Sénotier 1992). L'absence de qualification des emplois familiaux repose ainsi sur la similitude des tâches avec celles qui constituent le travail domestique gratuit.

Dans de nombreuses associations visitées, les responsables s'accordent pourtant à dire combien il leur est impossible d'envoyer n'importe quelle femme, du jour au lendemain, pour intervenir auprès de bénéficiaires, parfois impotents parce que malades, handicapés ou âgés, et par là en grande partie dépendants d'elles. Mais les processus de reconnaissance formelle qui transformeraient les qualités souhaitées en "qualification" sont pour l'instant encore largement absents.

La non-qualification se mesure donc au niveau de la rémunération, le plus souvent au salaire minimum, et aussi dans le fait que le seul diplôme existant dans ce domaine, le CAFAD (Certificat d'Aptitude à la Fonction d'Aide à Domicile) ne peut être obtenu que par la formation continue. Cela suppose donc un exercice préalable en l'absence de cette formation et annihile l'effet de reconnaissance dont il pourrait être porteur.

Ainsi, les emplois de proximité d'aide à la vie quotidienne apparaissent-ils comme bien éloignés de l'emploi salarié typique caractéristique ${ }^{13}$ des années de croissance économique. Cet éloignement s'accompagne d'un

13 ...que l'on peut rapidement décrire comme un emploi à durée indéterminée (CDI), à temps plein, à employeur unique, et présentant un minimum de qualification et de garanties sociales. Sur ce point, voir Puel (1980). 
"marquage féminin", puisque toutes les caractéristiques que l'on a pu montrer renforcent l'assignation des femmes et l'exclusion des hommes de ce type d'emploi. On peut donc s'interroger sur les risques d'enfermement qu'ils comportent pour les femmes concernées.

\section{L'EMPRISE DES LOGIQUES DOMESTIQUES}

L'objectif visé par le développement des emplois de proximité est de permettre à des chômeuses de retrouver, par l'emploi, une insertion sociale minimum. Mais on vient de voir que ces emplois sont, de manière générale, des emplois "atypiques", marqués au féminin. Dans quelle mesure alors permettent-ils à des femmes peu ou pas qualifiées, de s'intégrer sur le marché du travail salarié et d'acquérir une "professionnalitén? N'y a-t-il pas là des risques, du fait de la localisation domestique de ces emplois d'une relégation accrue de ces femmes dans cet espace et dans les relations qui le caractérisent?

\subsection{Des antécédents dans la domesticité et le bénévolat}

L'entrée des femmes des milieux populaires dans le salariat par le travail domestique n'est en effet pas nouvelle. Depuis le xixème siècle, les emplois de domestique ont représenté une des principales formes d'activité féminine salariée non agricole. Plus récemment, l'activité des travailleuses familiales s'est développée sous l'impulsion des associations familiales populaires catholiques ${ }^{14}$ pour soulager des tâches domestiques les mères de famille des milieux populaire en cas de maternité, de maladie ou d'hospitalisation. Ces deux formes de substitution au travail domestique gratuit ont donné naissance à deux modèles d'emploi fort différents, mais tous deux très éloignés des logiques de l'emploi salarié typique.

\subsubsection{Domesticité et servilité}

Le modèle de la "domesticitén s'en écarte, malgré son ancrage ancien dans le salariat, du fait du cadre d'exercice privé du travail, le logement de l'employeur, souvent même confondu avec le logement des domestiques, et des relations que cela laisse supposer entre domestique et pa-

14 Sur l'histoire de ces associations, voir Loiseau (1996). 
tron. Ces salariés pas comme les autres ont donc longtemps été tenus pour suspects par le mouvement ouvrier et ont eu les plus grandes difficultés à accéder au droit du travail ${ }^{15}$. Parce qu'ils restaient soumis à un large arbitraire de la part de leurs employeurs, leur liberté semblait entravée, dans leur propre vie privée même. Plus que leur simple travail, c'est donc l'intégralité de leur personne qui semblait avoir été aliénée par le contrat de travail, dans une relation personnalisée à leur employeur que l'on peut qualifier de "servile". Aujourd'hui, les "domestiques" sont devenues des "employées de maison", puis des "femmes de ménage". Les changements de vocabulaire reflètent des transformations importantes de situation. La domestique était généralement logée chez son employeur, qui l'utilisait dès lors à plein temps. La femme de ménage, elle, a généralement plusieurs employeurs différents chez lesquels elle n'effectue, on l'a vu, que quelques heures par semaine. Mais l'idée de sujétion et même de servilité attachée à ce modèle d'emploi est restée, que rejettent farouchement pourtant les femmes de ménage, refusant d'être "traitées comme des domestiques".

\subsubsection{Aide à domicile et bénévolat}

L'autre modèle est celui de al'aide à domicilen. A l'opposé du modèle de la domesticité, il est destiné à l'origine aux familles de milieu populaire. Il est issu de l'idée de charité chrétienne: les premières "aides à domicilen furent les religieuses des "Petites Souurs de l'Assomption" qui dès 1865 soignaient à domicile les malades et effectuaient en même temps les tâches ménagères. Ces religieuses seront ensuite relayées dans la $1^{\text {ère }}$ moitié du xxème siècle par des jeunes filles de la bourgeoisie qui accompliront ainsi de façon bénévole des tâches destinées à permettre le maintien d'un cadre propice à la cohésion des familles populaires. Enfin ce sont des associations de la mouvance catholique-sociale telles le MPF (Mouvement Populaire des Familles) ou encore l'ADMR (Aide à Domicile en Milieu Rural) qui créeront les premiers services de "travailleuses familiales". Ces mêmes organismes, ou leurs héritiers institueront ensuite des services d'aide à domicile, pour les cas où la dimension de "travail social" est moins importante, l'apport d'une aide étant nécessitée par l'état de santé du bénéficiaire plus que par sa situation sociale (c'est le cas en particulier des personnes âgées), qui les éloignent de leurs terrains d'intervention populaires traditionnels.

15 L'inspecteur du travail, par exemple, ne peut accéder à leur lieu de travail, qui est aussi un lieu privé, pour vérifier l'application du droit. Voir Martin-Huan (1997). 
On est ici aux antipodes du modèle de la domesticité. Le maître mot est a'aiden: les tâches domestiques ne sont envisagées que comme un support ${ }^{16}$ (certes irremplaçable) d'une action avant tout destinée aux personnes et encore plus à la sauvegarde de l'institution familiale. L'aide est donc plus d'ordre moral que matériel et cette action s'est longtemps accomplie sur le mode du bénévolat, ne semblant pouvoir s'envisager sur un mode salarié qu'avec beaucoup de réticences. Peut-on vraiment aider "pour de l'argent"?

Lors de la politique récente de promotion des emplois de proximité, ces deux modèles se sont trouvés confrontés entre eux. En effet la recherche de "gisements" d'emploi a conduit à privilégier la solvabilisation de la demande pour des services qui semblaient pouvoir être effectués par des demandeurs(ses) d'emploi peu ou pas qualifiés. Cela revenait à s'appuyer sur le modèle de la domesticité. Mais celui-ci n'étant guère revendiqué ni par les employeurs, ni par les salariées, c'est le modèle de l'aide qui a été mis au premier plan... en contradiction avec la forme même des emplois (le plus souvent de gré à gré). On assiste donc actuellement à des tiraillements entre les deux modèles, les associations d'aide à domicile tendant à insister sur la nécessaire "professionnalisation" de leurs salariées et à se démarquer ainsi d'une part des associations d'insertion dont l'objectif essentiel est de fournir une porte d'entrée dans l'emploi à des chômeurs (et ici essentiellement chômeuses) peu ou pas qualifiés et donc potentiellement "peu employables", d'autre part des relations d'emploi de gré à gré.

Du fait des modifications législatives des années récentes, s'est ainsi produit un brouillage entre des situations auparavant distinctes et à peu près stabilisées: celle des "femmes de ménage" d'une part, caractérisée par l'emploi direct par des particuliers, celle des aaides à domicile" d'autre part, caractérisée par l'emploi par une association ou une collectivité et la reconnaissance d'un minimum de qualifications. Aujourd'hui au contraire, certaines femmes de ménage sont employées par des associations (en particulier associations intermédiaires), tandis que des aides à domicile sont employées directement par des particuliers, même si elles dépendent du service "mandataire" d'une association ${ }^{17}$ (elles sont alors couvertes par la convention collective des employées de maison), elles peuvent parfois

16 Voir Bonamy (1997) qui explique l'importance du "faire" dans l'action sociale menée par les travailleuses familiales: elles gagnent en quelque sorte leur entrée (et la confiance) dans les familles grâce à leur activité ménagère.

17 Pour des précisions sur ces situations administratives, voir la synthèse particulièrement éclairante de Causse, Fournier et Labruyère (1998). 
occuper successivement les deux statuts et passer d'une convention collective à une autre dans une même journée!

Pourtant, malgré leurs différences et oppositions, ces deux modèles d'emploi se rejoignent au moins sur un point: ils se substituent à du travail domestique gratuit et ils tendent à se modeler sur les caractéristiques de ce dernier, s'éloignant par là même du rapport salarial typique pour se rapprocher d'un rapport social de type privé. Cela entraîne des difficultés particulières pour construire une véritable qualification de ces emplois et pour situer les relations qu'ils provoquent comme des arelations professionnelles" dans l'espace public.

\subsection{Les difficultés de construction de la qualification}

Le développement des emplois de proximité repose sur un paradoxe des objectifs de l'action publique. D'un côté, ces emplois ont été favorisés parce que, non qualifiés, ils permettaient d'embaucher sans délai des chômeurs non diplômés. D'un autre côté, l'objectif affiché par toutes les parties, qu'il s'agisse des pouvoirs publics ou des associations intervenant dans le domaine est la "professionnalisation" de ces emplois. Or la caractéristique première de tâches "professionnelles", quelle que soit la définition adoptée, réside dans les savoirs et savoir-faire spécifiques mis en œuvre. Parce que ces emplois se substituent au travail domestique gratuit et en conservent nombre de caractéristiques, ils ne semblent pas nécessiter ces pré-requis.

\subsubsection{Invisibilité et déqualification}

Le travail domestique gratuit des femmes chez elles est largement invisible. Transparent dans les statistiques, il est aussi invisible pour les acteurs masculins, seules les femmes semblant "voir ce qu'il y a à faire", ... et pour les femmes qui l'effectuent elles-mêmes ${ }^{18}$, permettant ainsi la perpétuation d'une répartition inégalitaire des tâches. Cette invisibilisation du travail se retrouve quand il est externalisé.

Il faut dire qu'une partie tout à fait essentielle du travail domestique est effectivement invisible. Elle est constituée par cet ensemble immatériel de prévision, de planification, d'organisation, de coordination sans

18 Ainsi que nous l'avons montré dans notre ouvrage (Dussuet 1997). Voir aussi Chabaud, Fougeyrollas et Sonthonnax (1985). 
lequel les tâches matérielles ne pourraient être accomplies, et qu'on peut désigner comme "préoccupation" (Dussuet 1997; Haicault 1984): ce type de travail aussi est attendu des salariées qui interviennent à domicile dans le cadre des emplois de proximité. Une "aide à domicile" compétente n'est pas seulement celle qui réalise correctement nettoyage, repassage, cuisine, etc, tâches qui lui sont expressément demandées, mais celle qui pensera à faire quelques provisions avant un week-end, qui s'ingéniera à faire participer à des tâches simples la personne âgée qui lui est "confiée", lui rendant ainsi un peu d'autonomie, souvent au prix d'une difficulté supplémentaire d'exécution pour elle-même. De la même manière, une bonne femme de ménage ne se borne pas à exécuter les tâches mais pense à faire aussi ce qu'on ne lui a pas demandé, sait s'organiser au mieux pour utiliser son temps d'intervention de la manière la plus efficace. Ces salariées ne sont donc pas, comme on le suppose un peu vite, du simple personnel d'exécution. Le fait d'être le plus souvent "seules" ${ }^{19}$, c'est à dire sans le contrôle (mais aussi sans le soutien) d'une hiérarchie et d'un collectif de pairs, les place dans la position de responsable de l'intégralité des tâches. Pourtant ce travail immatériel, parce qu'invisible, est le plus souvent nié dans son existence même, et n'est donc pas sanctionné par une reconnaissance de qualification.

Les tâches matérielles aussi sont souvent invisibilisées. N'est-ce pas seulement quand une maison est sale que "cela se voit?? Comme signalé précédemment, les interventions à domicile sont le plus souvent réclamées en dehors des temps de présence des utilisateurs, l'idéal semblant être un travail invisible. Nous retrouvons là une caractéristique essentielle requise des domestiques dans les maisons bourgeoises, la discrétion. Ces travailleuses intervenant dans l'espace privé de vie des utilisateurs ne peuvent apparaître que comme des facteurs de trouble si elles se font "remarquer"; leur intrusion dans l'espace intime n'est supportable que si elle est indécelable. Ainsi que le rapporte une responsable associative à propos d'utilisateurs:

Certains disent..., le mari dit: "OK pour une femme de ménage, mais...”. Y a des conditions aussi: «je veux pas la voir.

Se rendre invisible permet de réaliser le miracle de la "fée du logis", une expression à noter puisqu'elle signifie au fond que ce qui est réalisé par les femmes dans leur foyer relève de la magie, et pas du travail! Pour beaucoup de femmes, cela constitue un modèle puisqu'elles sont nom-

19 Comme le souligne le titre du livre de Brigitte Croff (1994). 
breuses à tenter de cacher leur propre travail domestique en le réalisant le plus possible en dehors de la présence de leur conjoint ou des enfants (Dussuet 1997). Les femmes de ménage ne peuvent véritablement remplacer cette "fée du logis" qu'en réalisant la même prouesse, c'est à dire en s'activant en dehors de la présence des membres de la famille et cela a comme conséquence la difficulté d'accéder au temps plein. Il faut noter que, dans les quelques cas d'exercice à temps plein rencontrés, le temps de travail est défini de façon plus extensive, intégrant les trajets entre lieux d'intervention, et souvent aussi des moments de coordination ou de formation continue. C'est dire qu'alors, le travail des salariées est défini autrement qu'en référence au travail domestique gratuit. Il acquiert alors une dimension "professionnelle" qui lui permet de sortir de la précarité et d'accéder à une certaine reconnaissance de qualification.

Invisible, le travail des femmes intervenant à domicile est donc le plus souvent nié dans son existence même, et par là, déqualifié. Mais ce déni n'est possible que parce que ce travail est identifié au travail domestique gratuit des femmes chez elles, qu'il n'est donc pas besoin d'en parler, de l'évoquer pour qu'il soit effectué. Il peut rester indéfini.

\subsubsection{Non-dit et déqualification}

La similitude des tâches avec celle du travail domestique gratuit permet en effet de se contenter d'en donner une description minimale et, en restant dans l'implicite, d'occulter les qualifications qu'elles nécessitent. Comme l'a montré Sarah Lecomte dans son étude des cadres statistiques et juridiques du groupe "employées de maison", la grille de classification de la convention collective fonde ses distinctions non sur les caractéristiques des tâches à effectuer, mais sur leur rôle social: elles sont présentées comme des "âches ménagères et familiales" (Lecomte 1996), sans plus de précision. Elles ne sont jamais décrites concrètement, ni clairement circonscrites, comme s'il s'agissait à chaque fois d'une évidence. Les intervenantes à domicile doivent "effectuer... les tâches ménagères courantes, les courses, certaines démarches administratives" (Fichier ROME). $\mathrm{Si}$ les "tâches ménagères courantes" sont détaillées en "ménage, vaisselle, lessive, repassage, rangement", on peut s'interroger sur ce que recouvre par exemple la tâche de "rangement", et que dire du "ménage"? La référence ici est implicitement celle d'une norme commune partagée. Le nondit est celui de l'évidence du sens commun. Tout le monde saurait très bien ce que l'on entend par "faire le ménage", la norme de la "maison propre et rangée" étant partagée par tous. 
Cela s'explique si l'on rappelle que la femme de ménage réalise un travail de substitution au travail domestique de la "maitresse de maison". Or ce travail-là, effectué gratuitement par la plupart des femmes, est aussi passé sous silence, tant il est évident. Parallèlement, il ne peut qu'être déqualifié. Non que ces tâches soient sans importance, nul ne songerait à minimiser le rôle de l'alimentation, de la propreté et de l'hygiène, des soins aux personnes, aux enfants tout particulièrement, mais aussi aux personnes âgées dépendantes. Mais, semble-t-il, elles ne s'apprennent pas, et c'est naturellement que les femmes savent les effectuer. Cela revient à dire qu'être femme suffit pour détenir une compétence dans ce domaine, mais exclut du même coup ces tâches de la sphère des apprentissages formels. Evidentes pour tout individu (femme) normalement constitué, elles seraient donc l'application du simple bon sens (féminin). Les tâches domestiques ne nécessiteraient pas de véritable formation, donc pas de qualification.

Ceci n'empêche pas toutefois de réclamer aux intervenantes des qualités garantes de leur "professionnalité. Dans leur discours d'auto-présentation, les associations d'aide à domicile insistent sur leur objectif de professionnalisation comme contradictoire avec l'embauche de n'importe quelle femme. Les tâches demandées, argumentent-elles, constituant un véritable service d'aide à la personne, ne sauraient être réalisées par la première demandeuse d'emploi venue: il faut au contraire mobiliser dans ces tâches de véritables professionnelles. Certains responsables d'association d'aide expliquent ainsi les difficultés qu'ils éprouvent à recruter des salariées, y compris dans une conjoncture de chômage important. Les demandeuses d'emploi envoyées par l'ANPE (Agence Nationale pour l'Emploi) apparaissent inadaptées aux exigences des tâches à effectuer. Il y a donc là un paradoxe. D'une part, les postes de travail sont décrits comme des postes d'exécution, non qualifiés; d'autre part, il semble qu'il faille pour les occuper des femmes "compétentes", faisant montre d'une véritable qualification professionnelle. Mais, là encore, il faut remarquer que les compétences requises ne se déclinent que sous la forme de listes de "qualités". Dans leur quête de critères, certaines associations ont rédigé des sortes de chartes internes qui présentent ce que l'une d'elles appelle par exemple "les règles d'or de l'aide à domicile». Les aides à domicile devraient ainsi ufaire preuve d'attention, de patience [...], de sens de l'organisation, de sang-froid, d'initiative, de dynamisme, de diplomatie, de tolérance, de discrétion, de ponctualité, d'honnêtetén. Malgré la taille impressionnante de cette liste, parce que ces "qualités" sont perçues comme fondées dans la sphère privée, parce qu'elles sont d'ordre moral, aucune "qualification" ne s'en dégage. Il est intéressant d'ailleurs de relever le glissement des ter- 
mes employés: on parle de "compétences" et non de "qualifications", seules ces dernières étant reconnues dans les conventions collectives.

C'est seulement quand les professions évoquées "Sortent" de la référence domestique, en participant au champ médical par exemple (comme c'est le cas pour les aides-soignantes, qui interviennent parfois aussi à domicile) que la notion de qualification, et donc une rémunération plus élevée apparaissent. Ainsi, plus les compétences exigées s'éloignent de qualités féminines perçues comme naturelles, et appartenant à la sphère privée, plus elles se dissocient de la personne, et semblent formalisables, nécessitant une formation, plus elles sont alors sources de qualification.

\subsection{Les difficultés à sortir du mode de relations domestique}

Les difficultés de construction de la qualification sont donc liées à l'emprise qu'exerce le monde domestique sur les représentations des compétences nécessaires au travail. D'une manière plus générale, on peut remarquer que le mode de relations propre à l'univers domestique tend à influencer le travail dans ces services de proximité d'aide à la vie quotidienne.

\subsubsection{Un travail personnalisé}

Ainsi par exemple est-il fort difficile aux salariées qui interviennent à domicile de distinguer dans leurs activité ce qui a qualité de travail et ce qui ressort du loisir. Pour les aides à domicile par exemple, le fait de passer du temps à discuter avec les personnes aidées peut être interprété comme une activité hors travail (voir sur cette question le point de vue des aides à domicile dans Juhel 1998), voire comme une perte de temps. Il est alors d'autant plus difficile de compter son temps de travail et de le limiter (problème que l'on trouve aussi dans le travail domestique des femmes chez elles). L'incertitude sur le travail débouche ainsi sur l'impossibilité de le cerner précisément et donc de le quantifier. Cela paraît contradictoire avec la nature du rapport salarial où la rémunération se présente comme la sanction d'une quantité précise de travail fournie. Dans une étude sur les relations entre aides-ménagères et personnes âgées, Brigitte Juhel (1998) montre comment certaines aides-ménagères font par exemple les courses des personnes âgées chez lesquelles elles interviennent sur leur propre temps libre, donc, au total, gratuitement.

Mais ces difficultés à définir le travail sont aussi l'indice d'une absence de séparation entre la personne de la travailleuse et le travail réalisé, l'in- 
dice, donc, d'un travail hautement "personnalisén. Ces femmes qui interviennent à domicile sont en effet censées remplacer le travail de la femme du foyer. Cela peut apparaitre comme une gageure: les tâches domestique ne se limitent pas à leur contenu concret: elles ont aussi une valeur hautement symbolique. Faire la cuisine ou le ménage, entretenir le linge, le repasser, c'est aussi instituer un ordre familial, c'est dire la nature du groupe familial, c'est l'ancrer dans une culture, le fonder socialement. Les manières de faire sont l'expression d'appartenances multiples qui situent le groupe familial en un point singulier de l'espace social. Il n'est donc pas indifférent que ces tâches soient réalisées par une personne ou une autre, par la fille, l'épouse, la mère... ou par une femme extérieure. Pourtant la demande des clients à l'égard des salariées intervenant à leur domicile repose sur cette ambiguité: elle présume une norme partagée, et rien n'est moins sûr que cette évidence-là. La diversité des manières de faire n'a d'égale que la rigidité de la norme qui les régit: d'un foyer à l'autre, les normes d'accomplissement des tâches domestiques varient considérablement, mais dans le même temps, chacune est persuadé que la norme qu'elle applique est une évidence commune à l'ensemble de l'humanité, civilisée du moins. Dès lors, le moindre écart peut être perçu comme une transgression insupportable. Le travail d'intervenantes extérieures en est rendu plus complexe: que faut-il faire, ou ne pas faire? comment le faire? La description des tâches à réaliser apparait comme une trivialité, mais, pour cette même raison, la non observation des normes implicites propres au foyer-client rejette l'intervenante dans l'animalité.

Cette exigence de personnalisation du travail se réalisait autrefois dans les familles bourgeoises par l'acculturation des domestiques, dont on pouvait dire qu'ils(elles) "faisaient partie de la famille.. Entrant souvent dès leur plus jeune âge à leur service, les "bonnes" pouvaient réaliser un travail parfaitement ajusté aux attentes de leurs patrons. Aujourd'hui encore, le travail à accomplir se situe bien loin de tâches standardisées et anonymes réalisables par des salariées interchangeables. Les associations employeuses le savent bien qui notent la difficulté d'imposer le changement ou le remplacement d'une salariée pour des périodes de formation ou tout simplement de congé.

Cette personnalisation est profondément ambivalente car, si elle est perçue comme souhaitable par les salariées qui y voient une valorisation de leur activité, la preuve qu'elles sont reconnues dans leur humanité, elle peut aussi se révéler dangereuse, entraînant la relation salariale sur la voie de la servilité. Il y aurait alors en effet confusion entre deux manières d'effectuer les mêmes tâches, l'une "professionnelle", l'autre "domestique. Car si les femmes salariées effectuent les mêmes gestes, exé- 
cutent les mêmes tâches que dans leur propre foyer, ce n'est pas pour autant du même travail qu'il est question. Quand elles les réalisent aen tant que fille, épouse ou mère, elles marquent en même temps leur place dans un ordre social et familial. En tant que salariée, ce travail symbolique peut se révéler nuisible en brouillant les statuts occupés. Il y a donc là, du fait de cette personnalisation des tâches, une difficulté à définir clairement le rapport entre employeur et salariée comme rapport salarial, le risque étant d'entraîner la salariée dans un rapport social de type privé qui engage plus qu'elle ne le souhaiterait sa propre personne.

\subsubsection{Une logique du don}

Dans leur propre foyer, le travail domestique effectué par les femmes ressortit à la logique du don telle que l'a analysée Marcel Mauss (1983): l'important ne se situe pas tant dans la matérialité des tâches effectuées que dans le fait que ce soient elles, les épouses, les mères, les filles qui les effectuent. L'essentiel est alors le lien institué plus que le service rendu. Cela explique que les règles de l'échange marchand ne s'appliquent pas à l'intérieur du foyer: l'équivalence entre les prestations de chacun n'est pas requise. Au contraire, un fonctionnement à̀ la dette ${ }^{20}$ permet l'entretien du lien familial.

Effectuer ce travail sous une forme salariée ne garantit pas la sortie de cette logique du don. On a déjà évoqué les difficultés pour les salariées à compter leur temps quand elles interviennent chez des personnes dépendantes avec lesquelles s'institue une relation personnalisée. Les aides à domicile ne peuvent alors refuser un service, pourtant extérieur à la définition de leur travail comme salariée, à ceux qu'elles arrivent à considérer comme des membres de leur famille. De tels comportements peuvent aussi être observés chez les femmes de ménage qui cherchent parfois, en donnant plus que ne le prévoit le contrat, à aller au delà de la simple relation salariale. Faire le "petit plus" qui permet de montrer que l'on s'investit dans son travail, que l'on n'est pas une machine est aussi ce qui permet de se faire reconnaitre par le client. Ainsi Mme P., femme de ménage, qui a pris soin des plantes sans qu'on le lui demande a pu instaurer grâce à cela des relations différentes avec sa cliente:

c'est plus agréable, j'ai une dame, qui travaille maintenant parce qu'elle est professeur, pendant l'été, elle était là, elle venait me voir, on discutait, elle me de-

20 Ainsi que le montrent Françoise Bloch et Monique Buisson (1998) dans l'exemple de la garde des enfants. 
mandait des conseils pour les plantes, elle en a même parlé à sa voisine, "faudra demander des conseils à Mme P. , elle s'y connaît, et quand j'arrivais, elle me disait "vous allez boire un petit café avec nous" alors je buvais le café et on discutait avant de travailler... je trouve que c'est bien.

En même temps, ce glissement vers la privatisation de la relation est un danger que certaines ressentent bien: comment refuser alors un aservice" supplémentaire? Cette tendance est donc ambivalente car les salariées y trouvent une manière de se faire reconnaître comme "personne humaine", de faire valoir leur qualités personnelles, mais le risque est évidemment dans l'exploitation de leur attitude par des employeurs peu scrupuleux ou plus simplement négligents de la réglementation.

Cette dérive est favorisée par la forme d'emploi de gré à gré où, à l'inverse de la situation typique qui confronte un employeur à de multiples salariés, la salariée se trouve seule face à de multiples employeurs. On peut alors observer des arrangements entre soi qui aboutissent souvent à spolier les salariées de droits qu'elles ne connaissent pas toujours (périodes de congés de l'employeur qui se traduisent par un nonemploi, demande d'heures supplémentaires à la dernière minute, etc), même si elles y trouvent parfois aussi des avantages (faire varier l'horaire d'arrivée ou de départ en fonction de leurs propres contraintes personnelles par exemple).

La question de savoir qui perd ou qui gagne à l'échange ne se pose de toute façon pas puisque, dans la logique du don, la relation elle-même est à la fois le support et la fin de celui-ci. En mettant entre parenthèses les catégories du jugement marchand, cette logique explique à la fois la non-qualification, l'invisibilité et la personnalisation du travail que l'on a observées ci-dessus: l'objectivation et le calcul concernant les prestations échangées y sont inenvisageables puisqu'ils signifieraient leur mise en balance, l'établissement d'un bilan, donc un arrêt de l'échange,... et de la relation. La distanciation avec cette logique est d'autant plus difficile à opérer que les salariées intervenant à domicile pénètrent dans le cadre privé de l'habitation, là où la limite avec l'intime est la plus difficile à situer. Cela contribue à qualifier les relations qui s'y nouent comme "privées", alors même que la relation salariale appartient clairement à la sphère "publique.. Dès lors, s'introduit une incertitude sur les règles de justice (au sens de Boltanski et Thévenot 1991) applicables: sont-elles d'ordre domestique et privé, auquel cas, la logique du don fonctionne (on peut bien faire quelques heures supplémentaires qui entreront dans le système de don-contre-don et seront reconnues, par exemple, dans l'ordre de l'affectif), ou bien d'ordre salarial et public, et c'est l'ordre marchand qui règne (pas question alors de réaliser un travail sans contrepartie). 
Dans ces emplois, le rapport marchand de vente de la force de travail tend donc à être euphémisé. Les formes d'emploi qui se sont développées ces dernières années, où les salariées elles-mêmes ne savent plus très bien qui est leur employeur véritable (associations mandataires agissant pour le compte de particuliers-employeurs par exemple), accentuent encore cette tendance, les rapports hiérarchiques d'autorité/subordination se distinguant alors du rapport employeur/salariée.

Les emplois de proximité d'aide à la vie quotidienne présentent donc, pour les femmes qui les occupent, un certain nombre de risques de relégation dans la sphère domestique, du fait de l'emprise des logiques domestiques sur la constitution de ces emplois. La situation inégalitaire qu'elles connaissent tant dans la famille que dans l'espace public repose sur une division sexuelle du travail établie dans un rapport social de sexe. Au sein de la famille, c'est "en tant que femmen, que les filles, les épouses, les mères réalisent l'essentiel du travail domestique. Elles le font parce qu'elles le conçoivent comme une partie intégrante de leur rôle féminin, dans un rapport social qui n'est pas salarial (elles ne vendent pas leur force de travail) mais "domestique", dans une logique du don. Pourtant, en accomplissant ces tâches, parce qu'elles sont le lieu d'un rapport social de sexe, les femmes subissent une assignation au domestique qui est un mécanisme essentiel de leur domination. Même salariés, des emplois construits sur ce modèle domestique ne peuvent avoir d'effet d'émancipation, ni d'intégration dans la société globale.

\section{LE RÔLE DES ASSOCIATIONS}

La caractérisation privée ou publique, domestique ou salariale du rapport social engagé dans les emplois de proximité est donc un enjeu est de taille pour les femmes. Ce n'est qu'en mettant à distance les tâches domestiques, en les réalisant comme un véritable "travail", extérieur à elles-mêmes qu'elles sortiraient du rapport social domestique. Quel rôle peuvent alors jouer les associations? A quelles conditions peuvent-elles opérer la "professionnalisation" qu'elles annoncent comme un objectif et éviter aux femmes employées quelques-uns des risque que nous venons de décrire? Toutes les associations ne sont pas forcément conscientes des enjeux mais leur caractère non-lucratif, les objectifs qu'elles se sont donnés les empêchent d'ignorer totalement le problème de la qualité des emplois qu'elles contribuent à créer.

Nous nous intéresserons ici à plusieurs exemples, pas forcément représentatifs de toutes les structures associatives, mais pas exceptionnels 
non plus, d'associations qui tentent de construire des dispositifs de professionnalisation des emplois.

\subsection{Les enjeux de la formation}

On a vu plus haut les difficultés à construire de la qualification là où les "qualités féminines" semblent suffire pour détenir la compétence nécessaire à l'emploi. Pourtant, les différentes associations rencontrées s'accordent pour reconnaître, à des degrés divers, la nécessité d'une formation.

\subsubsection{Le CAFAD: une formation facultative et mal reconnue}

Les associations d'aide à domicile tout d'abord, sont les plus réticentes à embaucher "n'importe qui" pour intervenir chez leurs "bénéficiaires", qui sont pour la plupart des personnes âgées, handicapées ou malades, ou encore des familles en difficulté. Leurs responsables, souvent anciennes bénévoles, militent depuis longtemps pour la reconnaissance de la "profession d'aide à domicile" et disent toutes la difficulté de l'exercice d'une profession qui oblige à côtoyer des situations de détresse physique et morale. La demande est réelle et affirmée d'une formation destinée à permettre une meilleure prise en charge des différentes pathologies que les salariées sont amenées à rencontrer, qu'il s'agisse d'alcoolisme, de maladies nerveuses, de SIDA, ou tout simplement de dépendance physique due à l'âge. Plus discrète est par contre la demande d'une formation spécifique aux techniques des tâches domestiques qui sont le lot quotidien des salariées, comme si cette formation-là n'était pas légitime. Sans doute faut-il y voir la marque de la difficulté qu'ont les "aides à domicilen à se dissocier de l'image de la "femme de ménagen à laquelle elles sont le plus souvent assimilées.

Les responsables encouragent donc leurs salariées à se former pour obtenir le CAFAD (Certificat d'Aptitude à la Fonction d'Aide à Domicile), mais elles savent que ce diplôme ne leur permettra pas d'améliorer leur situation matérielle (pas de prise en compte au niveau du salaire). La conception de cette formation, non obligatoire pour exercer, et accessible seulement aux salariées en emploi (dès lors forcément embauchées sans le diplôme), avec une formule d'alternance, ne favorise pas la reconnaissance souhaitée. Les budgets de formation de ces associations sont en tout cas insuffisants pour permettre à toutes les salariées l'accès à ce diplôme, qui ne joue donc pas un grand rôle dans la construction d'une profession d'aide à domicile. 


\subsubsection{Qualification, tâches domestiques, tâches industrielles}

C'est donc, de façon un peu paradoxale, plutôt vers les associations d'insertion qu'il faut se tourner pour observer cet effet de la formation. En effet celles-ci, plus que les associations d'aide orientées avant tout vers la satisfaction des besoins des personnes aidées, sont attentives à la qualité des emplois qu'elles proposent à leurs salariées. Il est donc intéressant de voir que plusieurs d'entre elles ont élaboré des formations internes destinées aux salariées qu'elles "accompagnent".

Le premier exemple est celui de deux associations qui commercialisent des services d'entretien du linge et spécialement de couture, effectués dans un atelier et une boutique. Il s'agit bien d'externalisation de travail domestique, car longtemps ces tâches ont été effectuées à domicile par la mère de famille. Mais avec la commercialisation d'articles de confection industrielle à bas prix, la couture tend à disparaître des activités domestiques ${ }^{21}$. Les associations éprouvent donc certaines difficultés à trouver dans le public en insertion qui leur est adressé des femmes qui détiennent ces savoir-faire, que la famille semble transmettre de moins en moins. Elles offrent donc parfois des emplois que seules des titulaires de CAP, de BEP ou même de diplômes supérieurs paraissent capables de tenir. Ces emplois sont ainsi d'emblée constitués comme "qualifiés" et les associations sont obligées de construire une formation minimum sur le tas par des "encadrantes techniques", souvent très qualifiées, dont le parcours familial justifie le retrait de la production marchande industrielle et qui, par leur présence même, démontrent la qualification des emplois proposés.

On voit bien dans cet exemple comment la construction de la qualification est liée à l'éloignement du monde domestique et au rapprochement avec celui de l'entreprise industrielle. L'option du travail réalisé en atelier contribue à ce rapprochement, en situant clairement ce travail hors du monde domestique.

\subsubsection{Une formation obligatoire}

Le second exemple est celui d'une association d'insertion qui ne propose pourtant que des emplois de "femme de ménage", c'est à dire des emplois considérés comme les moins qualifiés parmi l'ensemble de ces

21 Le temps moyen quotidien en est passé de 18 à 9 minutes entre 1986 et 1999 , selon la dernière enquête budget-temps de l'INSEE: Brousse (1999). 
emplois de proximité. Mais l'association a posé comme principe que "ce n'est pas parce qu'on sait faire le ménage chez soi qu'on sait le faire chez les autres". Les conséquences de cette proposition sont importantes puisqu'elle signifie qu'on peut "savoir faire chez soì et devoir apprendre à faire chez les autres.

Faire accepter cette idée aux femmes à la recherche d'un emploi est d'autant plus difficile que celles-ci estiment le plus souvent que cette compétence "domestique" est justement la seule chose qu'elles ont à monnayer sur le marché du travail. Une proportion importante de femmes renâclent donc devant ce qu'elles considèrent comme un obstacle supplémentaire à leur insertion. Mais l'association ayant rendu cette étape de formation obligatoire, les femmes de ménage que nous avons pu rencontrer ont toutes suivi cette formation. A posteriori elles se montrent toutes satisfaites d'avoir effectué ce stage, même si, pour plusieurs d'entre elles, il a dû être accompli bénévolement, l'association n'ayant pas toujours trouvé les moyens financiers pour les rémunérer pendant cette période. Quand on cherche à inventorier avec elles les acquis de cette formation, elles mettent en avant les aspects les plus techniques, les plus "visibles": apprendre à se servir d'une centrale à vapeur de repassage, d'un appareil à nettoyer les vitres, savoir distinguer les différents types de fours et leur mode de nettoyage. Elles expriment par exemple leur satisfaction de disposer de "fiches techniques" concernant telle ou telle tâche précise:

On avait pas mal de feuilles sur tous les fours, les micro-ondes, les nouveaux fours, tout ça, oui, toutes les feuilles qu'ils nous donnaient, ça c'était bien, bien expliqué et tout (Mme E).

Les responsables, eux, ont une autre approche: ils soulignent d'abord que leur démarche part des compétences pratiques réelles des femmes, de leur expérience, leur intervention de formateur consistant souvent en une explicitation de ces compétences et une incitation à l'échange à l'intérieur du groupe de stagiaires.

On part de ce qu'elles savent faire, on part de ce qu'elles savent et après on apporte des rectificatifs, parce qu'elles savent faire des choses, hein, beaucoup, mais elles savent pas... elles en ont pas forcément conscience.

Mais ils insistent surtout sur les apprentissages d'ordre comportemental, sur les aspects les plus immatériels de cette formation: la présentation de soi, les savoirs relationnels, la prise en main de l'organisation du travail leur paraissent plus essentiels dans la réussite de l'insertion des femmes concernées dans leurs futurs emplois que les savoirs proprement techniques: 
Réfléchir, s'organiser, organiser son poste de travail, aussi par rapport à sa santé, se préserver quoi... c'est pas on se lance dans le ménage à fond, on transpire...

On peut interpréter cette discordance dans la perception de la formation en remarquant que ce ne sont pas les gestes du ménage qui font défaut à ces femmes: le plus souvent, elles les réalisent très bien chez elles, dans leur propre foyer. Même si l'équipement ménager des foyers employeurs est souvent plus sophistiqué, elles n'utilisent pas quotidiennement les techniques plus complexes apprises au cours du stage. Ces techniques ne sont donc sans doute pas vraiment fondamentales dans la réalisation de leur travail comme salariée, mais elles le sont en tant que signe. Elles apparaissent comme un indice tangible de qualification (puisque résultat d'un apprentissage formel). Les apprentissages privilégiés par les responsables, sans doute plus importants pour la reconnaissance de cette qualification par les utilisateurs des services ne peuvent, faute de matérialité, constituer ce signe.

Ces deux aspects de la formation sont donc inséparables et contribuent à faire émerger un troisième volet, moins souvent cité, mais qui a sans doute aussi son importance: la constitution d'un collectif de travail. En effet, les femmes de ménage seront ensuite amenées à travailler en solitaires chez des particuliers. Le temps du stage de formation constitue alors un moment fondamental pour l'établissement de relations avec les autres salariées et les femmes interviewées ont souvent montré une certaine nostalgie à l'égard de ce qui apparaît comme un moment-clé de socialisation. Une socialisation multidimensionnelle qui doit s'entendre non seulement comme un temps d'apprentissage professionnel mais aussi comme une construction d'identité professionnelle (Dubar 1992) et une acculturation à ce qui pourrait devenir une "culture d'entreprise" où, plutôt ici, d'association.

Le temps de la formation est donc important non seulement par les savoirs et savoir-faire qu'il permet d'acquérir mais aussi parce qu'en signifiant que le sexe n'est pas en soi suffisant pour les détenir, il dissocie les compétences des femmes de leur personne.

\subsection{De l'implicite... à l'explicitation: la définition du ménage}

On a vu comment la déqualification reposait aussi sur le caractère implicite des tâches demandées. C'est ce "non-dit" qui identifie le travail de l'aide à domicile à celui de la femme de ménage, mais aussi, de la bonne à tout faire ou de la domestique. Il leur faut "faire... tout ce qu'il y a à fairen. Dans le cas des femmes de ménage employées sans intermé- 
diaire par des particuliers, la définition du travail est ainsi laissée à l'appréciation exclusive de l'employeur qui achète finalement des heures de présence, du temps, sans que l'on puisse vraiment préjuger de son utilisation.

\subsubsection{Un inventaire...}

Lors de l'emploi par une association, ce schéma peut être modifié. C'est toujours le cas avec les associations d'aide, dans la mesure où elles répondent souvent à une demande qui émane d'institutions (établissements hospitaliers qui s'apprêtent à faire sortir leur patient, Caisses de retraite, etc...) dont dépend plus ou moins le financement de la prestation. Les responsables visitent alors le domicile de l'utilisateur pour déterminer avec lui ou avec ses proches les tâches à effectuer. Cela permet de déterminer le nombre d'heures nécessaire d'intervention et d'engager leur financement, mais cela permet aussi de poser un accord sur "ce qui doit être fait".

Une association d'insertion a la même pratique. Ses responsables s'obligent à une visite préalable au domicile du futur client et tentent d'expliciter ce qui n'est habituellement jamais dit à propos du travail domestique: quelle fréquence de tâche est nécessaire? faudra-t-il repasser telle ou telle catégorie de vêtements, les plier? Ces responsables nous ont dit, et les salariées aussi, la difficulté d'ajuster ensuite précisément le temps "vendu" à la demande. Cette difficulté est encore le résultat de l'invisibilité du travail domestique. Comment mesurer le temps nécessaire pour "faire à fond une piècen? La superficie, la nature des surfaces à nettoyer, le degré d'encombrement apportent des éléments d'appréciation mais l'essentiel reste inquantifiable, parce que le temps passé dépend à la fois du degré d'engagement du salarié dans la tâche et de l'exigence de propreté du demandeur. La tâche des responsables associatifs consiste donc aussi à bâtir un ensemble de normes de références, même si celles-ci restent toujours discutables: qu'est-ce que "faire le ménage"?

on évalue...on commence à évaluer, parce que...on fait tout parce qu'y avait rien avant, tout ce qui est ménage, repassage, on fait ça chez soi mais pas de la même façon, donc on commence à construire des outils: chemise, repassage...une cuisine à fond, on met tant de temps à peu près si il y a tel élément, surface, tant de mètres carrés à l'heure, on essaie de connaitre, de chronométrer. Mais ça dépend de la femme de ménage.

La demande des clients repose sur l'évidence présumée d'une norme partagée. Mais qu'en est-il réellement? Le ménage ne peut jamais être 
"parfait", il n'est donc jamais vraiment «terminé". Et même la perfection n'est pas forcément l'objectif visé. Certains utilisateurs trouvent que les femmes de ménage en font trop!:

ça dépend de l'exigence des clients... y en a qui disent "on aime mieux la qualité, qu'elle y mette du temps", y en a qui disent aussi: "mais elle va trop à fond dans les coins, c'est pas ça que je demande", alors on rectifie, c'est pas facile... on essaie de réajuster.

Malgré ces difficultés, l'intervention des associations permet que les choses soient dites et ainsi d'instituer une référence qui, même non écrite, est opposable aux exigences des utilisateurs: ce qui doit être fait est... ce qui a été dit.

\subsection{2. ...qui pose des limites}

Les femmes qui ont une expérience antérieure d' "employée de maison" (comme elles disent) expriment très clairement comment cette explicitation institue des limites à un travail qui, comme le travail domestique gratuit, a tendance à occuper tout le temps disponible. L'explicitation, en introduisant une distinction entre ce qui est à faire et ce "dont on n'a pas parlé" limite la tendance à l'extension de la tâche à ce "tout faire" qui est la règle du travail domestique et que l'on retrouve dans l'expression abonne à tout faire. Pour "l'employée de maison", la pression directe de la nécessité s'exerce sans médiation. Parce qu'on lui a confié non une tâche, qui connaît une fin, un achèvement, mais une fonction, "tenir la maison" (qui, elle, n'en connaît pas), elle ne peut légitimement s'arrêter de travailler que quand elle atteint ses propres limites physiologiques. A la différence de l'employée de maison, la femme de ménage peut ainsi compter son temps. En posant des bornes aux tâches à effectuer, l'explicitation effectuée par l'association permet aussi de limiter le temps à leur consacrer.

Employée de maison, c'est plus dur, parce qu'employée de maison, vous êtes, vous êtes toute seule du matin au soir, vous êtes obligée d'assumer la maison, moi, j'avais des patrons, ils partaient à $4 \mathrm{~h}$ 4-5h du matin, ils faisaient les marchés, moi, j'avais 6 enfants en garde, c'est à vous d'organiser le... de faire les chambres, de faire le ménage, la cuisine, la vaisselle, repasser, c'est à vous d'assumer jusqu'au soir, et puis ils rentraient pas de bonne heure, $9 \mathrm{~h}$, ou $10 \mathrm{~h}$ du soir, surveiller si les petits sont pas malades... Employée de maison, c'est encore plus dur qu'être femme de ménage, parce que femme de ménage, vous avez déjà moins de responsabilité, vous avez le ménage, la poussière, tandis que employé de maison, quand vous avez les enfants, il faut surveiller les enfants, appeler le 
médecin s'ils sont malades, c'est plus dur employée de maison, hein parce que là vous... y avait toute la charge de la maison sur le dos... et les courses, les... tout ça, faut... oui y avait les courses, préparer à manger, vous faites manger les enfants, après faut refaire à manger parce que les patrons ils arrivent du marché, faut refaire à manger, faut refaire la vaisselle (Mme C).

On voit sur cet exemple l'incidence de la forme prise par l'emploi sur l'extension des tâches à effectuer: la situation de "domestique", logée par son employeur, permet au travail de s'étendre d'une façon quasi illimitée. Le passage à la situation de "femme de ménage" suppose non seulement une dissociation du lieu de travail et du domicile, mais encore l'éventualité de l'intervention de la salariée chez d'autres employeurs. Tout cela concourt à la nécessité d'expliciter le contenu du travail attendu, et ce faisant, de l'objectiver. Mais la présence de l'association, qui facilite cette explicitation, contribue aussi à en garantir la validité. En cela elle joue un rôle important de médiation.

\subsection{L'association comme intermédiaire: une "publicisation" de la relation}

Dans les emplois «à domicilen, la figure classique d'emploi est celle de l'emploi direct par des particuliers qui paient un salaire horaire. Elle se distingue ainsi des situations idéales-typiques du salariat où l'employeur est généralement confronté à plusieurs salariés, dans une relation qu'on peut caractériser comme "publique. A l'inverse, la relation d'une femme de ménage avec son employeur se réduit le plus souvent à un "tête à têten, certes encadré par des règles juridiques issues du droit du travail et de la convention collective, mais dont le caractère "privé" et personnalisé, l'emporte généralement. Il suffit pour s'en convaincre de se référer aux multiples arrangements "entre soi" conclus par exemple entre les employeurs et leurs femmes de ménage qui aboutissent le plus souvent à spolier cellesci de droits qu'elles ne connaissent pas toujours.

Les associations interviennent donc comme des tiers dans cette relation. Cette entrée d'un "témoin" dans une relation au départ "privée" a pour effet de "publiciser" cette relation.

\subsubsection{Une relation "publique"}

Tout d'abord, le respect des règles légales est rendu nécessaire par le recours à l'association: pas question évidemment de rogner sur les congés payés ou "d'oublier" de payer les charges sociales. Le rôle des asso- 
ciations mandataires dans l'aide à domicile par exemple est ici considérable, car les particuliers, employeurs réels de bonne foi mais souvent peu conscients de leurs engagements, sont soumis, de par leur âge élevé, à des risques qui engagent leur rapports avec leur salariée. Ainsi, dans le cas d'une hospitalisation qui rend le service de l'aide à domicile inutile, une procédure de licenciement doit être engagée. La présence de l'association oblige à respecter ces règles salariales.

Mais la médiation de l'association va parfois plus loin: elle peut intervenir, on l'a vu, sur la définition des tâches à effectuer. Plus encore, en explicitant la demande des employeurs, elle contraint ceux-ci à faire un tri, une sélection dans ce qui est "demandable", ou pas, dans le cadre d'une relation salariale. En cas de litige, les salariées n'hésitent guère, semble-t-il à faire intervenir les responsables de l'association, qui sont ainsi érigés en juges: qu'il s'agisse de réévaluer le temps nécessaire pour réaliser les tâches demandées, ou qu'il s'agisse de refuser des tâches vécues comme humiliantes. Cela conduit parfois l'association à rompre le contrat avec certains clients ayant une conception que les responsables considèrent comme dépassée de la relation salariale, ne correspondant pas aux normes des relations "publiques". Par exemple, pour cette responsable d'une association d'insertion proposant les services de femmes de ménage, cette cliente

demandait beaucoup trop, beaucoup trop exigeante, quoi, elle était encore au xixème siècle, vraiment, la bonne, la servante, donc, c'est pas ça qu'on veut, quoi.

Mais la médiation peut jouer aussi en faveur de ménages utilisateurs déçus par le travail réalisé. Citons l'exemple d'une femme de ménage en insertion dont les soucis personnels avaient abouti à une dégradation sensible du travail. Les particuliers utilisateurs ont alerté l'association, un peu gênés de perturber ainsi une démarche d'insertion dans laquelle ils se sentaient partie prenante. L'intervention de l'association a alors permis de "dépersonnaliser" la situation et, ce faisant, de la dédramatiser en l'extrayant d'un cadre privé pour en faire un conflit public, au sens où les normes en vigueur dans l'espace public sont applicables ${ }^{22}$ :

on dit les mots, on dit aux clients: "on peut vous proposer quelqu'un d'autre et puis on va rencontrer la personne», quand c'est comme ça, on téléphone au reste

22 On peut analyser cette opération comme une manière de déplacer le travail effectué par les femmes de ménage d'une sphère de la justice edomestique "à une sphère de la justice "civique", selon la classification des "cités" de la justice opérée par Luc Boltanski et Laurent Thévenot (1991). 
de clients; on intervient, et là, il s'avère que ça se passait mal, et qu'ils n'osaient pas téléphoner, ils savaient pas comment nous dire que ça se passait mal...

L'association apparaît ainsi comme le garant du caractère public de la relation, et en tant que tel, elle est l'agent d'une production normative définissant l'extension respective du public et du privé.

\subsubsection{L'intime: privé du domestique}

Cette définition s'avère particulièrement fondamentale dans ces emplois qui ont pour cadre l'espace privé des utilisateurs. La médiation de l'association permet alors de délimiter, au cas par cas, un espace de relations public à l'intérieur même du cadre privé du logement. Les limites de ce "public du privé" sont celles entre l'intime et le privé montrable, celles entre le "personnel" et le "domestique" proprement dits. Mais elles pourraient bien être aussi celles définissant le travail salarié en opposition au travail "servile", comme le montre par exemple le refus des responsables d'une association de répondre à la demande de clients désirant faire laver à la main leurs sous-vêtements par l'employée:

Les deux cas où y a eu des problèmes avec les clients, c'est des clients qui donnaient leur linge à laver à la main, des sous-vêtements, des machins..., dans les 2 cas, elles les considéraient comme leurs bonnes, il fallait qu'elles lavent leurs bas à la main, leurs soutien-gorge, slips,... là, ça passe pas.

Dans cette tâche de lessive à la main du linge corporel ${ }^{23}$, se jouait un contact entre les corps beaucoup trop intime pour qu'il soit acceptable, selon cette responsable associative, dans une relation salariée. Demander ce travail-là à une personne étrangère au cercle familial, c'était demander "une bonne", obliger cette personne à une relation engageant sa propre intimité, donc sortir de la relation salariale type, pour rentrer dans une relation de domesticité.

Les responsables interviewés sont pourtant lucides sur le caractère culturel et par conséquent fluctuant de cette limite qu'ils ont eu eux-mêmes l'occasion de vérifier. Par exemple, pour certaines femmes de ménage d'origine étrangère, la frontière ne se situe pas au même point que pour les Françaises puisque certaines refusent catégoriquement de repasser des sous-vêtements d'homme pourtant propres, ... tâche somme toute

23 Sur les rapports entre l'entretien du linge et la souillure corporelle, voir Denèfle (1995). 
acceptable au regard de la norme française contemporaine. Dans cette affaire, l'important est sans doute moins dans la place de la limite, soumise à l'arbitraire culturel, que dans son existence. Affirmer l'existence d'une limite, quelle qu'elle soit, c'est dire que tout n'est pas possible, c'est donc situer la relation et le travail effectué dans un cadre normé, institué par les règles de l'espace public.

A cet égard, les différences sont importantes entre les associations. Certaines servent seulement d'intermédiaires entre des demandeuses d'emploi et des utilisateurs: le contrat de travail reste alors un accord entre deux personnes privées, facilement soustrait aux normes publiques. Quand les associations sont employeuses, elles effectuent plus souvent, mais pas toujours, ce travail de "publicisation", essentiel dans le processus de "professionnalisation".

Il reste pourtant une question essentielle: celle de savoir quel peut être l'avenir des femmes engagées dans ces emplois. L'entrée dans l'espace public qu'elles ont parfois ainsi opérée correspond-elle à une insertion durable? Quelles sont leurs possibilités d'évolution, de promotion? Peuvent-elles espérer construire une véritable "carrière professionnelle" à partir de ces emplois leur permettant de faire valoir des compétences acquises dans la sphère domestique? Même si les évolutions que nous venons d'évoquer sont trop récentes pour répondre de façon définitive à ces questions, on pressent que le rôle des associations pourrait ici encore se révéler crucial. C'est en effet par leur organisation, par les relations qu'elles établissent entre elles qu'elles pourraient dessiner de véritables filières professionnelles, permettant de passer de la place de simple afemme de ménage", à celle "d'aide à domicile", voire de "travailleuse familiale". Cela suppose des systèmes de formation directement liés à l'emploi et une collaboration réelle des différentes structures. Des mouvements dans ce sens sont esquissés, par exemple sur l'agglomération d'Angers, avec la création d'une association regroupant la quasi-totalité des association angevines offrant des services à domicile, dans un objectif de coopération. Mais cela supposerait aussi d'éclaircir les rapports entre les différents "métiers" de cette filière, ce qui apparaît difficile à réaliser, les uns ayant tendance à se définir par l'exclusion des autres.

Les emplois de proximité d'aide à la vie quotidienne apparaissent ainsi comme un lieu de brouillage des rapports sociaux. Plutôt qu'un point d'entrée des femmes dans la relation salariale, ils représentent actuellement une transposition, dans le cadre salarié, d'un rapport "domestique, rapport social de sexe impliquant au delà de l'échange de force de travail, l'établissement de relations personnalisées entre la travailleuse et ses employeurs-utilisateurs. D'une certaine manière, ils déplacent la frontière 
entre espace privé et espace public, en faisant du rapport salarial établi un rapport de type privé. Dans ce sens, ils peuvent constituer de véritables pièges en enfermant une fois de plus les femmes concernées dans des positions et statuts sociaux définis par leur sexe. Ils ne contribueraient alors qu'à reproduire les inégalités dont elles sont victimes tant dans l'espace public que dans l'espace privé.

Mais ce processus n'est pas une fatalité. A la condition de se situer clairement dans la sphère du salariat, ces mêmes emplois peuvent être une source d'émancipation, comprise comme "individuation", en permettant aux femmes d'accéder à des relations de type public. On voit alors l'importance des acteurs qui mettent en forme ces emplois, les associations en tout premier lieu. Si elles réalisent effectivement le travail de médiation permettant de sortir de l'invisibilité du travail et de la logique du don, si elles réussissent à engager un réel processus de qualification en mettant en lumière le travail effectué, elles pourraient extraire ces emplois des relations de domesticité, et procurer aux femmes des milieux populaires une opportunité de professionnalisation.

\section{BIBLIOGRAPHIE}

Bailly, Jean-Paul. 1996. Le développment des services de proximité. Rapport. Conseil Economique et Social. Paris: Direction des journaux officiels.

Bloch, FrANÇOISE et MONIQUE BuISSON. 1998. La garde des enfants, une bistoire de femmes. Entre don, équité et rémunération. Paris: L'Harmattan.

Boltanski, LuC et LAURENT THÉVENOT. 1991. De la justification: les économies de la grandeur. Paris: Gallimard.

Bonamy, Bernardette. 1997. Les défis de la travailleuse familiale. Paris: Erès.

BROUSSE, CÉCILE. 1999. "La répartition du travail domestique entre conjoints reste très largement spécialisée et inégale", France, portrait social 1999-2000: 135-151. Paris: INSEE.

Causse, lise; Christine fournier et Chantal labruyère. 1998. Les aides à domicile, des emplois en plein remue-ménage. Paris: Syros.

CÉAlIs, RozA. 1997. “Le devenir des salariés des associations intermédiaires". Premières informations et premières synthèses 33, 2. Paris: DARES.

CÉAlis, RozA et SERGE Zilberman. 1997. "Les emplois familiaux et les organismes de services aux personnes en 1996". Premières informations et premières synthèses 46, 1. Paris: DARES.

Chabaud, Danièle; Dominique Fougeyrollas et Françoise Sonthonnax. 1985. Espace et temps du travail domestique. Paris: Méridiens.

CHADEAU, ANN. 1992. "Que vaut la production non-marchande des ménages?.. Revue économique de l'OCDE. Paris: OCDE.

CHADEAU, ANN et ANNIE FOUQUET. 1981a. "Peut-on mesurer le travail domestique?.. Economie et Statistique 136. Paris: INSEE. 
- 1981b. Le travail domestique, essai de quantification. Archives et Documents. Paris: INSEE.

CONSEIL D'ANAlyse eCONOMIQue. 1998. Les emplois de proximité. Paris: La Documentation Française.

Croff, BRIGITTE. 1994. Seules, genèse des emplois familiaux. Paris: Métailié.

Dalla Costa, Mariarosa et Selma James. 1973. Le pouvoir des femmes et la subversion sociale. Genève: Librairie Adversaire.

DeNĖFLe, SylVETTE. 1995. Tant qu'y aura du linge à laver. Paris: Arléa-Corlet.

DUBAR, ClaUdE. 1992. La socialisation, construction des identités sociales et professionnelles. Paris: Colin.

DUSSUET, ANNIE. 1997. Logiques domestiques - essai sur les représentations du travail domestique chez les femmes salariées de milieu populaire. Paris: L'Harmattan.

FiCHIER ROME. s. a. (Répertoire Opérationnel des Métiers et Emplois). ANPE.

GADREY, JEAN. 1996. Services: la productivité en question. Paris: Desclée de Brouwer.

GrefFE, XAVIER. 1990. Nouvelles demandes, nouveaux services. Commissariat général au Plan. Paris: La Documentation Française.

GUILBERT, MADELEINE. 1996. Les fonctions des femmes dans l'industrie. Paris: Mouton.

HaICAUlT, MONIQuE. 1984. "La gestion ordinaire de la vie en deux". Sociologie du travail 3 .

JePSEn, MARIA et DANIÈLE MEULDERS. 1998. "Are local employment initiatives able to generate quality employment? The impact on female labour force. Tansfer 4, 3 (Bruxelles: European Trade Union Institute).

JUHEL, BRIGITTE. 1998. L'aide ménagère et la personne âgée, petites et grandes manoeuvres autour d'un espace de vie à partager. Paris: L'Harmattan.

KERBOURCH, JEAN-YVES. 1997. Les groupements d'aide aux personnes, approche juridique. Série Observatoire, document $n^{\circ}$ 121. Marseille: CEREQ.

Kergoat, Danièle; Françoise Imbert; Hélène le Doaré et Danièle SÉnotier. 1992. Les infirmières et leur coordination. Paris: Lamarre.

LECOMTE, SARAH. 1996. Les employées de maison: cadrage bistorique, juridique et statistique - Histoire d'un groupe professionnel aux contours flous. Mémoire de DEA sous la direction de Joëlle Deniot. Université de Nantes.

LOISEAU, DOMINIQUE. 1996. Femmes et militantisme. Paris: L'Harmattan.

MARTIN-HUAN, JACQUELINE. 1997. La longue marche des domestiques, en France, du XIXè siècle à nos jours. Nantes: Opéra Editions.

MARUANI, Margaret (dir.). 1998. Les nouvelles frontières de l'inégalité - Hommes et femmes sur le marché du travail. Paris: La Découverte.

MAUSS, MARCEL. 1983. "Essai sur le don", Sociologie et anthropologie: 143-279. Paris: PUF. PuEL, Hugues. 1980. "Il y a emploi et emploin. Travail et emploi 4. Paris: Ministère du Travail.

Zilberman, SeRge. 1995. L'évolution des emplois familiaux de 1992 à 1994. Paris: DARES Premières synthèses n. 109. 